\title{
Programmable Porous Polymers via Direct Bubble Writing with Surfactant-Free Inks
}

\author{
Dahlia N. Amato, Douglas V. Amato, Michael Sandoz, Jeremy Weigand, Derek L. Patton,* \\ and Claas Willem Visser*
}

Cite This: ACS Appl. Mater. Interfaces 2020, 12, 42048-42055

Read Online

ACCESS

Llll Metrics \& More

Article Recommendations

Supporting Information

ABSTRACT: Fabrication of macroporous polymers with functionally graded architecture or chemistry bears transformative potential in acoustic damping, energy storage materials, flexible electronics, and filtration but is hardly reachable with current processes. Here, we introduce thiol-ene chemistries in direct bubble writing, a recent technique for additive manufacturing of foams with locally controlled cell size, density, and macroscopic shape. Surfactant-free and solvent-free graded three-dimensional (3D) foams without drying-induced shrinkage were fabricated by direct bubble writing at an unparalleled ink viscosity of $410 \mathrm{cP}$ (40 times higher than previous formulations). Functionalities including shape memory, high glass transition temperatures

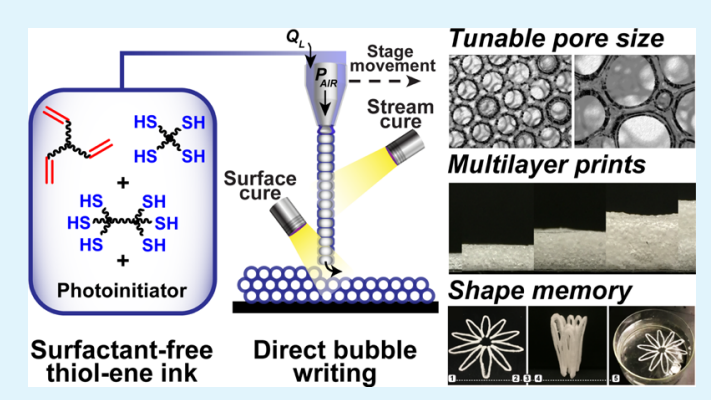
$\left(>25{ }^{\circ} \mathrm{C}\right)$, and chemical gradients were demonstrated. These results extend direct bubble writing from aqueous inks to nonaqueous formulations at high liquid flow rates $\left(3 \mathrm{~mL} \mathrm{~min}^{-1}\right)$. Altogether, direct bubble writing with thiol-ene inks promises rapid one-step fabrication of functional materials with locally controlled gradients in the chemical, mechanical, and architectural domains.

KEYWORDS: polymer foams, 3D printing, nonaqueous foam, thiol-ene, functionally graded materials

\section{INTRODUCTION}

Solid polymeric foams constitute a widely applied material class with applications in cushioning (shoes, sofa, bed), ${ }^{1}$ oil sequestration, ${ }^{2}$ energy storage, ${ }^{3}$ robotics, ${ }^{4}$ pressure sensing, ${ }^{5,6}$ and scaffolds for artificial tissues. ${ }^{7-9}$ The widespread use of foams lies in their exceptional performance as compared to bulk materials, such as excellent weight/stiffness ratios, low heat transfer, permeability, and load-bearing potential by a single construct. ${ }^{1,10}$ Compared to natural foams, such as bone and bamboo, synthetic foams possess multiple shortcomings including poor control of the three-dimensional (3D) pore arrangement, outer shape complexity, and, especially, gradients in pore size, density, stiffness, and other relevant properties that would enable the integration of multiple functions by a single foam construct. ${ }^{10-12}$ Conventionally, synthetic foams rely on the introduction of air by mechanical action or chemical addition, resulting in a broad distribution of the cell size, that is, polydisperse foams. In recent years, monodisperse foams have been typically made in a two-step process, by first depositing monodisperse solids/emulsions in a matrix, and then removing the deposited material. ${ }^{13-17}$ While these approaches provide monodisperse foams with either open- or closed-cell architectures, ${ }^{7}$ the requirement of surfactant, limited throughput (typically a few $\mathrm{mL} \mathrm{h}^{-1}$ ), use of multiple precursor liquids, and multistep processes inhibit their immediate industrial viability. ${ }^{15,18}$ Additionally, integrating complex $3 \mathrm{D}$ geometries ${ }^{19-24}$ and a range of porosities within a single construct is still challenging. ${ }^{25}$ A simple, robust, and scalable technique to produce surfactant-free foams with defined cellular size/structure and 3D cell arrangement with a broad access to aqueous/nonaqueous monomers and minimal shrinkage/postcuring would therefore represent a major step forward. ${ }^{26}$

Direct bubble writing is a new printing technique that relies on the rapid solidification of bubbles upon impact on a substrate to ensure high fidelity printing. ${ }^{6}$ To print bubbles, pressurized gas is injected through the core of a core-shell nozzle with a coflowing outer shell fluid (aqueous or organic inks) to produce gas-filled bubbles with tunable size and porosities.

In our previous work, we demonstrated that acrylate-based direct bubble writing possessed gas-type dependent cellular structure (i.e., nitrogen $=$ closed-cell or air $=$ open-cell), locally tunable foam properties (density/modulus) via control over the bubble size/distribution, and the ability to print 3D lattices, shells, and out-of-plane pillars. ${ }^{6}$ Key benefits of this process

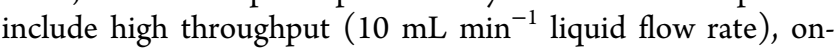

Received: April 30, 2020

Accepted: August 10, 2020

Published: August 10, 2020 

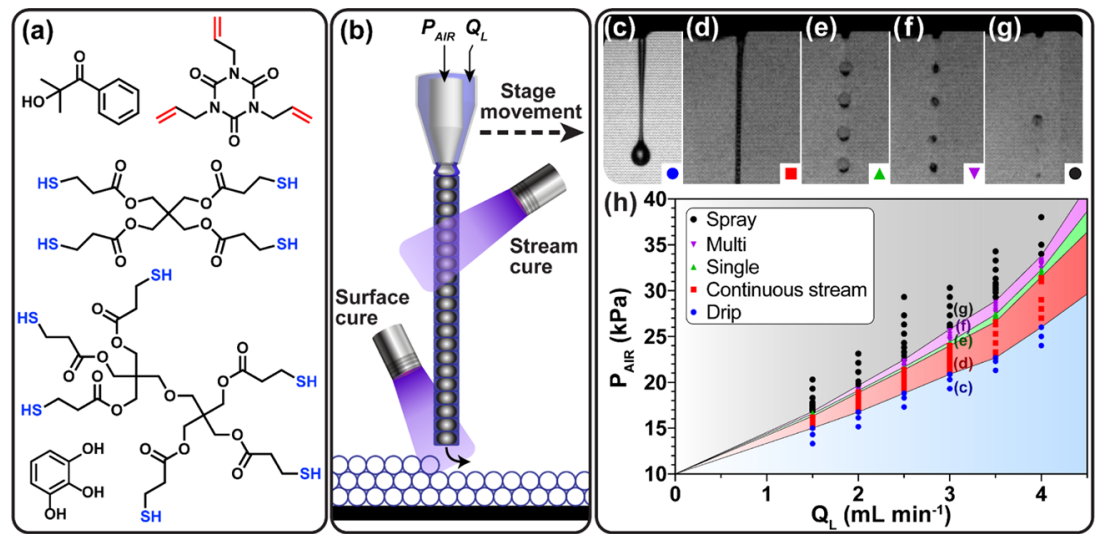

Figure 1. Overview of direct bubble writing. (a) Chemicals used in the ink formulation. (b) Schematic illustration of the printing process in which air coflows with the ink to produce a stream of bubbles, which is cured on-the-fly and after impacting the surface. (c-g) Resulting flow regimes observed at a constant ink flow rate $\left(Q_{\mathrm{L}}=3 \mathrm{~mL} \mathrm{~min}^{-1}\right)$ and varied air pressures: $(\mathrm{c})$ dripping $\left(P_{\text {air }}=16 \mathrm{kPa}\right)$, (d) continuous bubble stream $(21$ $\mathrm{kPa}),(\mathrm{e})$ monodisperse bubble formation $(24 \mathrm{kPa})$, (f) polydisperse multibubble formation (24 kPa), and (g) spraying (29 kPa). (h) Diagram depicting the $P_{\text {air }}$ and $Q_{\mathrm{L}}$-dependent fluid dynamics of surfactant-free foams.

the-fly tunable cell size/architecture, simple setup, and minimal clogging. However, direct bubble writing suffers from substantial shrinkage ( $28 \%$ in each dimension), use of surfactant for bubble stabilization, a limited monomer selection, and a prolonged drying step to remove the solvent (water, forming 59\% of the initial weight). To overcome these issues, we advance direct bubble writing to a solvent-free thiol-ene platform. Thiol-ene photopolymerization ${ }^{27-34}$ is insensitive to oxygen, does not need a surfactant, has negligible shrinkage, requires minimal postcure processing, allows for rapid postpolymerization modification, and enables access to higher glass transition temperature materials $\left(T_{\mathrm{g}}>23{ }^{\circ} \mathrm{C}\right)$. Such a robust platform provides tunable chemical/mechanical properties with simple "click" chemistries, further decreasing the barrier to applying the direct bubble writing platform.

\section{METHODS AND MATERIALS}

Materials. All reagents were obtained commercially and used without further purification unless otherwise specified. Triallylisocyanurate, pyrogallol, pentaerythritol tetramercaptopropionate (PETMP), dipentaerythritol hexakis(3-mercaptopropionate) (DiPETMP), and 2,2-dimethoxy-2-phenylacetophenone (DMPA) were purchased from Sigma-Aldrich. Darocure 1173 was donated by iGM Resins.

Printing Ink. Pyrogallol $(0.1 \mathrm{~g}, 0.79 \mathrm{mmol})$ serves as a radical inhibitor to stabilize the ink formulations ${ }^{35}$ and is first dissolved into triallylisocyanurate $(40 \mathrm{~g}, 0.16 \mathrm{~mol})$, followed by pentaerythritol tetramercaptopropionate (PETMP, $29.4 \mathrm{~g}, 0.06 \mathrm{~mol}$ ), dipentaerythritol hexakis(3-mercaptopropionate) (DiPETMP, $31.4 \mathrm{~g}, 0.04 \mathrm{~mol}$ ), and $4.2 \mathrm{~g}$ of the photoinitiator Darocure 1173 as shown in Figure 1a. Inks containing excess thiol were prepared similarly but with a $1.2: 1$ thiol-to-alkene ratio by increasing amounts of DiPETMP ( $41.9 \mathrm{~g}, 0.54$ mol) and PETMP (39.2 g, $0.08 \mathrm{~mol})$.

3D Printing Setup. Printing resins are loaded into $30 \mathrm{~mL}$ of HSW soft-ject luer-lock syringes and mounted into a NE-8000 New Era Pump Systems syringe pump. The loaded syringe is connected to the shell nozzle of a custom core-shell nozzle (for details, see ref 6 ) by a $1 \mathrm{~mm}$ diameter, $48 \mathrm{~cm}$ long tubing. Pressurized air connects to the core of this nozzle, resulting in the directed ejection of resin bubbles into air as depicted in Figure 1b (photographs of the setup are provided in Figure S1). The bubble train is deposited onto the print surface, where the resin is photopolymerized by ultraviolet (UV) light. The alignment of the UV light and the bubble deposition location is ensured by mounting the print nozzle and two fiber-optic light guides to a custom machined aluminum bracket, which is bolted to a LulzBot
TAZ 6 3D printer. Print paths for each geometry are written as Gcode scripts and loaded onto the 3D printer via an SD card. Air pressure is controlled via a Matlab-controlled Alicat Scientific electronic pressure controller, and resin flow is controlled via the syringe pump feed rate. UV light intensity for each light guide is controlled independently via two OmniCure light sources. One light source (OmniCure S2000) cures a stream of air-infused resin $7.5 \mathrm{~mm}$ from the nozzle tip at an angle of $18^{\circ}$ from horizontal. This precuring does not fully cure the ink and is designated as a "stream cure". The other light source (OmniCure S1000) cures the stream $78 \mathrm{~mm}$ from the nozzle tip at $70^{\circ}$ from horizontal, and it is designated as "surface cure". Printing parameters studied include air pressure $(0-40 \mathrm{kPa})$, print speed $\left(30-100 \mathrm{~m} \mathrm{~s}^{-1}\right)$, resin flow rate $\left(1.5-4.0 \mathrm{~mL} \mathrm{~min}^{-1}\right)$, and UV-cure intensity $\left(55.4-621 \mathrm{~mW} \mathrm{~cm}^{-2}\right)$. Unless otherwise indicated, each sample was printed with a constant resin flow rate of $3 \mathrm{~mL} \mathrm{~min}^{-1}$ and air pressure of $23 \mathrm{kPa}$ with a surface cure intensity of $30.6 \mathrm{~mW}$ $\mathrm{cm}^{-2}$ and a stream cure intensity of $301 \mathrm{~mW} \mathrm{~cm}$. The printing speed for each sample was varied using G-code commands to print 30 $\mathrm{mm}$ long sections for each printing speed. After printing, the samples were postcured with unfiltered UV light before being removed from the printing surface. Printed parts were exposed for $30 \mathrm{~s}$ by unfiltered UV light within $5 \mathrm{~min}$ after printing to ensure that the samples were thoroughly cured.

UV Intensity Study. The influence of UV-cure intensity on resin stream width and print quality was studied by independently varying stream and surface UV-cure intensity. Two experimental conditions were used: the first varying stream cure intensity with a constant surface cure intensity of $30.6 \mathrm{~mW} \mathrm{~cm} \mathrm{~cm}^{-2}$ and the second varied stream cure intensity with a constant surface cure intensity of $106 \mathrm{~mW} \mathrm{~cm}^{-2}$. The samples were then studied using a microscope to determine the print quality at each cure intensity.

Mechanical Testing. The influence of air pressure on foam mechanical properties was studied via uniaxial compression testing using a MTS 810 hydraulic test frame. Printing paths and speeds for each sample were controlled using G-code commands to print $50 \times$ $50 \times 10 \mathrm{~mm}^{3}$ blocks. From these blocks, compression samples were trimmed to $5 \times 5 \times 10 \mathrm{~mm}^{3}$ to remove edge effects and ensure the faces contacting compression platens were flat and parallel. The samples were compressed to a strain of $50 \%$ at a constant cross-head speed of $50 \mathrm{~mm} \mathrm{~min}^{-1}$ using a $100 \mathrm{kN}$ load cell. No fewer than four samples were tested at each air pressure.

Rheology. Ink formulations were evaluated with an ARES-G2 rheometer from TA Instruments (New Castle, DE) equipped with a UV light guide accessory. Following a $14 \mathrm{~s}$ equilibration period, the samples were irradiated by UV light from an unfiltered OmniCure S2000 UV light source via a fiber optic cable for $30 \mathrm{~ms}$. Unfiltered UV light intensity was calibrated at the sample location using an external 


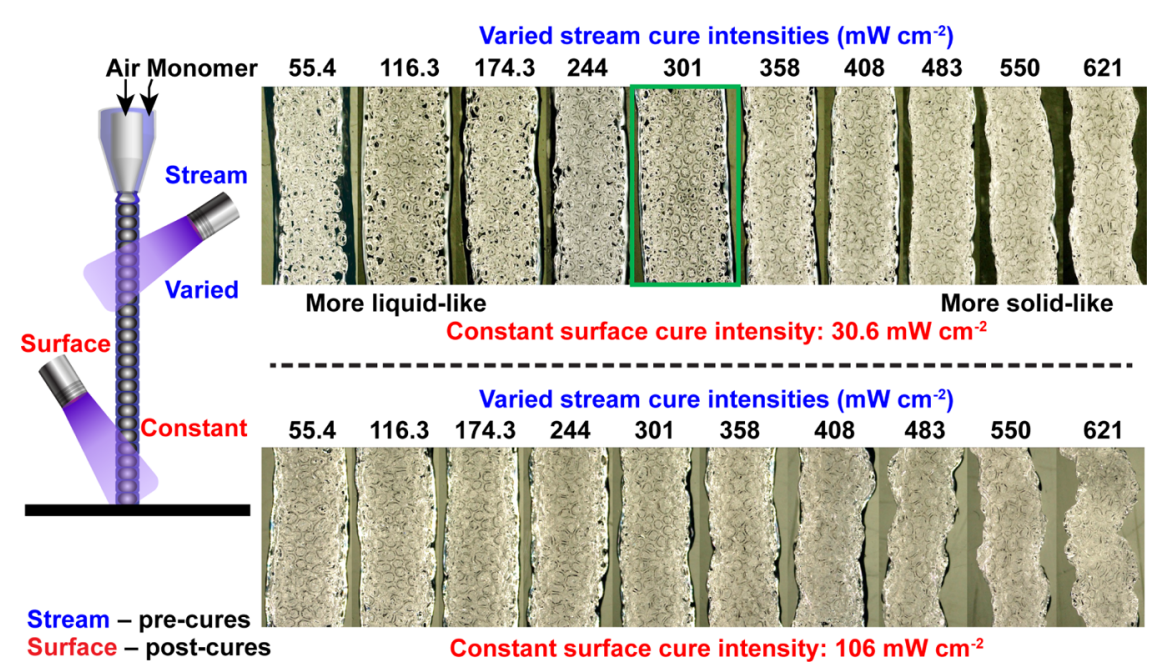

Figure 2. Photographs of printed filaments. Both the stream UV intensity (left to right) and the surface intensity (top-bottom) were varied as indicated.

radiometer. Photorheology experiments were conducted by loading samples between an aluminum (bottom) and quartz (top) $20 \mathrm{~mm}$ diameter parallel plates with a sample thickness of $0.5 \mathrm{~mm}$. Experiments were conducted using automatic strain adjustment at $10 \mathrm{~Hz}$ in "fast sampling" mode to resolve rheological behavior at fast cure speeds and to prevent over torque of the instrument. The viscosity was determined as a function of the shear rate with a stainless steel $25 \mathrm{~mm}$ cone and plate geometry $(0.1 \mathrm{rad})$ at $25^{\circ} \mathrm{C}$. The samples were presheared at $10 \mathrm{~s}^{-1}$ prior to steady shear experiments to ensure homogeneous samples before monitoring steady-state shear viscosity.

Postpolymerization Modification. Printed porous filaments prepared from inks containing $20 \mathrm{~mol} \%$ excess thiol were postmodified via thiol-ene and thiol-Michael reactions. For thiolene modification, the thiol-functional filaments were submerged in a tetrahydrofuran (THF) solution containing fluorescein $o$-methacrylate $(0.025 \mathrm{~g}, 0.06 \mathrm{mmol})$ and DMPA $(0.01 \mathrm{~g}, 0.04 \mathrm{mmol})$ and irradiated with $4 \mathrm{~mW} \mathrm{~cm}^{-2} \mathrm{UV}$ light for $30 \mathrm{~min}$ to ligate the fluorescein dye to the excess thiol surface. For thiol-Michael modification, thiolfunctional filaments were submerged in either an aqueous solution of Texas Red C2 maleimide ( $5 \mathrm{mg}, 0.007 \mathrm{mmol}$ ) or a THF solution of 1-[4-(trifluoromethyl)phenyl]-1H-pyrrole-2,5-dione $\left(\mathrm{CF}_{3}\right.$-MA, 0.025 g, $0.1 \mathrm{mmol}$ ) containing triethylamine for $12 \mathrm{~h}$.

Characterization. X-ray photoelectron spectroscopy was performed using a Thermo Fisher ESCALAB Xi ${ }^{+}$spectrometer equipped with a monochromatic Al X-ray source $(1486.6 \mathrm{eV})$. Measurements were performed using the standard magnetic lens mode and charge compensation. The base pressure in the analysis chamber during spectral acquisition was at $3 \times 10^{-7}$ mbar. Spectra were collected at a takeoff angle of $90^{\circ}$ from the plane of the surface. The pass energy of the analyzer was set at $150 \mathrm{eV}$ for survey scans with an energy resolution of $1.0 \mathrm{eV}$; the total acquisition time was $220 \mathrm{~s}$. Binding energies were calibrated with respect to C $1 \mathrm{~s}$ at $284.8 \mathrm{eV}$. All spectra were recorded using the Thermo Scientific Avantage software; data files were translated to VGD format and processed using the Thermo Avantage package v5.9904. Polymerization kinetics were measured by real-time Fourier transform infrared (FTIR) spectroscopy using a Nicolet 8700 FTIR spectrometer with a KBr beam splitter and MCT/ A detector. Each sample was exposed to a UV light with an intensity of $100 \mathrm{~mW} \mathrm{~cm}^{-2}$ using an Omnicure Series 1000 light source. Series scans were recorded at approximately 2 scan s ${ }^{-1}$ with a resolution of 4 $\mathrm{cm}^{-1}$. Thiol conversion was monitored by integrating the peak at $2557 \mathrm{~cm}^{-1}$ over time,

\section{RESULTS AND DISCUSSION}

To use direct bubble writing without both solvent and surfactant, a library of thiol and alkene monomers were probed for their ability to foam through a core-shell nozzle. While typical monomers have viscosities less than $10 \mathrm{cP}$, we found that higher viscosity monomers (90-2500 cP) still provide highly controlled bubble trains. The final ink formulation had a viscosity of $410 \mathrm{cP}$ and is comprised of hexa- and tetra-functional thiol monomers along with a trifunctional alkene and a photoinitiator (see Figure 1a). To study the influence of formulation changes to ink curing, different photoinitiator concentrations were evaluated in $30 \mathrm{~ms}$ pulse-UV rheometer experiments (Figure S2a). The rate at which the ink's complex viscosity builds after UV exposure monotonically increased as a function of the Darocur 1173 concentration, which was varied from 0 to $4 \%$. The $4 \%$ Darocur 1173 concentration reached a complex viscosity greater than $20000 \mathrm{cps}$ in less than $0.4 \mathrm{~s}$. Additionally, photoinitiators Darocur 1173 and SpeedCure TPO-L (2,4,6trimethylbenzoyldi-phenylphosphinate) were evaluated at different cure intensities $(2,30,60 \mathrm{~mW}$, with a $30 \mathrm{~ms}$ pulse, at 4 wt \%) (Figure S2b). Clearly, the light intensity had a greater effect than the photoinitiator type, highlighting the importance of light exposure intensity and location. Darocur 1173 was chosen for the final formulation since this photoinitiator consistently yielded higher viscosity materials compared to the TPO-L.

To probe the bubble ejection regime from the core-shell nozzle for this highly viscous ink, we systematically varied the flow rate of the liquid resin mixture $\left(Q_{L}\right)$ and the air pressure $\left(P_{\text {air }}\right)$. Figure $1 \mathrm{~b}$ depicts the experimental setup of the nozzle configuration to study bubble ejection and simultaneously print $3 \mathrm{D}$ structures. As shown in Figure $1 \mathrm{c}-\mathrm{h}$, at all flow rates tested $\left(1.5 \leq Q_{L} \leq 4.0 \mathrm{~mL} \min ^{-1}\right)$, five distinct ejection mechanisms were observed. At low $P_{\text {air }}$, pure-liquid resin jetting/dripping occurred (Figure 1c). Here, the gas is unable to overcome the threshold pressure required to enter the liquid as previously described. ${ }^{6}$ As $P_{\text {air }}$ increases, the length of the jet increased until a critical point where small bubbles become continuously entrained within a jet of resin to form a connected bubble stream (Figure 1d). A further increase in $P_{\text {air }}$ leads to a transition away from filaments and to the production of disconnected monodisperse bubbles (droplets with a liquid shell and a gas core; Figure 1e). As $P_{\text {air }}$ is again further increased, multibubble ejections (Figure 1f) and 
eventually random spraying occurs (Figure $1 \mathrm{~g}$ ). To afford the highest control and minimize defects during printing, we selected a flow rate $Q_{L}$ of $3 \mathrm{~mL} \mathrm{~min}^{-1}$ for all experiments. Higher flow rates are also well-controlled but result in too short print times for our syringe-fed setup.

Multiple configurations of the UV light guides were evaluated (i.e., a single stream/surface light or double stream/surface lights). Interestingly, if both fiber-optic lines are positioned in the stream cure position, the UV-curing of this connected bubble stream leads to the production of porous fibers, which at present cannot be printed in a controlled fashion. Alternatively, when just a single light source was used (stream or surface), the ink was allowed to flow too much, leading to poor retention of the porous structures. The combination of a stream light with a surface light allowed the ink to flow enough for higher-fidelity layer-by-layer printed structures. In Figure 2, we determine the optimal curing conditions of the bubbles by varying the intensity of the stream curing from 55 to $621 \mathrm{~mW} \mathrm{~cm}^{-2}$ at two different surface cure

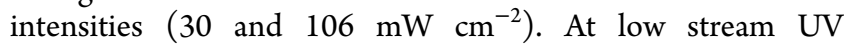
intensities $\left(55-174 \mathrm{~mW} \mathrm{~cm}{ }^{-2}\right)$, the ink that impacts the substrate is able to flow, leading to regions of high resin density and regions with bubbles. This result is readily explained by the reduction in curing, allowing time for the resin to separate from the bubbles prior to polymerization as seen in the 55.4 $\mathrm{mW} \mathrm{cm} \mathrm{cm}^{-2}$ printing condition. At medium cure intensities (244-408 $\mathrm{mW} \mathrm{\textrm {cm } ^ { - 2 }}$ ), the bubbles are distributed over the entire filament and a more uniform structure was observed (similar thickness throughout the printed line with uniform bubble placement). At $483-621 \mathrm{~mW} \mathrm{~cm}^{-2}$ and at higher surface cure intensity $\left(106 \mathrm{~mW} \mathrm{~cm}^{-2}\right)$, a tortuous printed line was observed. We assume that the stream was cured immediately upon impact with the surface, such that capillary forces were prevented from relaxing the internal surfaces (the bubble air-resin interface) as well as the external surfaces (smoothening the edges of the filament). Eventually, $30.6 \mathrm{~mW}$ $\mathrm{cm}^{-2}$ surface cure with $301 \mathrm{~mW} \mathrm{~cm} \mathrm{~cm}^{-2}$ stream cure was selected for all experiments moving forward.

To print objects with multiple layers, the influence of print speed on the resulting line width (Figure 3a) and the layer thickness as a function of the number of layers (Figure $3 b$ ) were systematically studied. To assess the line width, filaments were formed by laterally moving the printhead in the $x-y$ direction at programmed print speeds. For printing speeds of $V$ $>20 \mathrm{~mm} \mathrm{~s}^{-1}$, continuous filaments are formed with decreasing filament width as the velocity of the printhead is increased (Figure 3a). As the ejected filaments are cured in situ, they can be stacked to produce 3D architectures. As Figure 3b shows, the total thickness $\left(L_{\mathrm{T}}\right)$ increases linearly $\left(1.4 \mathrm{~mm} \mathrm{layer}^{-1}\right)$ with the number of applied layers $\left(L_{\#}\right)$, indicating that the local impact and solidification process are robust with respect to the height of the construct.

With the ability to print consistently, we examined the influence of the gas pressure on the resulting cell size, foam density, and Young's modulus. As the gas pressure $\left(P_{\text {air }}\right)$ increases from 21.7 to $22.6 \mathrm{kPa}$, the average bubble diameter (DB) increases from $107 \pm 8$ to $173 \pm 15 \mu \mathrm{m}$, respectively (Figure $4 \mathrm{a}$ ). The coefficient of variance $(\mathrm{CV})=\sigma / \mathrm{m}$ was determined, where $\sigma$ is the standard deviation and $m$ is the mean cell size as detailed in Figure S3. In the monodisperse ejection regime, a tight distribution of $4.9 \%<\mathrm{CV}<6 \%$ was achieved. This is an improvement compared even to the recent microfluidic techniques for which $\mathrm{CV} \approx 20 \%$ was reported as
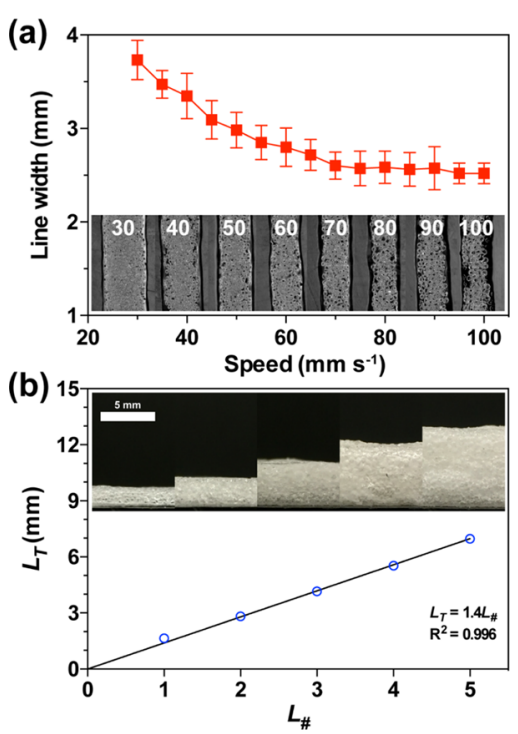

Figure 3. Filament width and layer height. (a) Line width is shown as a function of the lateral velocity of the printhead. The inset shows photographs of the printed filaments at different velocities. (b) Total height of the printed construct $\left(L_{\mathrm{T}}\right)$ as a function of the number of layers $\left(L_{\#}\right)$.
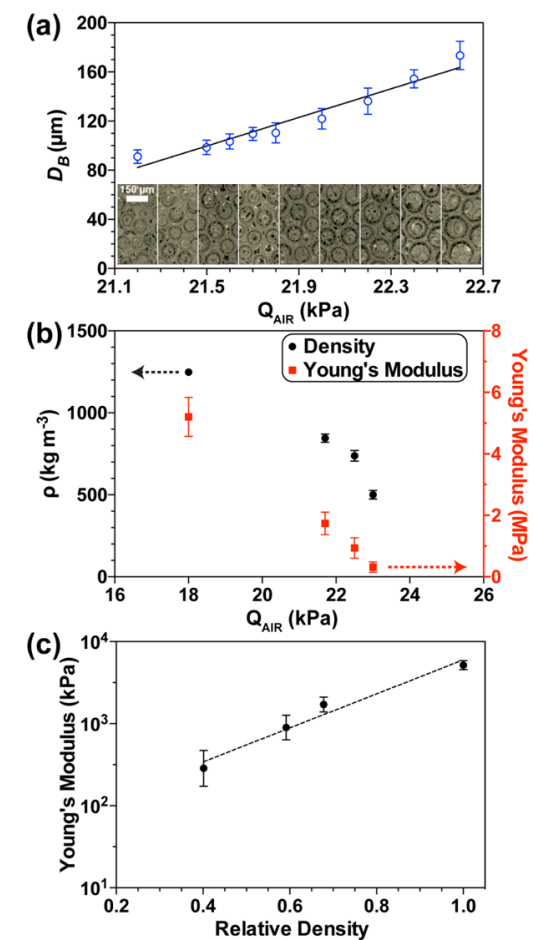

Figure 4. Tuning the porosity and mechanical properties of printed porous solids at a constant ink flow rate $\left(Q_{\mathrm{L}}=3 \mathrm{~mL} \mathrm{~min}^{-1}\right)$. (a) Value of the cell diameter as a function of $P_{\text {air }}$ (b) Density and Young's modulus as functions of $P_{\text {air }}$ (c) Young's modulus as a function of relative density. The dashed line is a guide to the eye with a slope of $3.2 \pm 0.1$.

the state of the art for solid foams. ${ }^{36}$ Figure $4 \mathrm{~b}$ shows that increasing the pressure also decreases the density of the foams from $845 \pm 25 \mathrm{~kg} \mathrm{~m}^{-3}$ at $21.7 \mathrm{kPa}$ to $501 \pm 26 \mathrm{~kg} \mathrm{~m}^{-3}$ at 22.6 $\mathrm{kPa}$. It is well-known that the density of foams controls their stiffness. ${ }^{37}$ Figure $4 \mathrm{c}$ shows Young's modulus $Y$ as a function of the density, revealing tunability over more than an order of 
magnitude (from $0.32 \pm 0.17$ to $5.5 \pm 0.7 \mathrm{MPa}$ ). The dashed line in Figure $4 \mathrm{c}$ shows the exponent $\alpha$ of the relationship $Y \sim$ $\rho^{\alpha}$, where $\rho$ is the relative density. The observed value $\alpha=3.2$ exceeds the literature value for both open-cell foams $(\alpha=2)$ and closed-cell foams $(\alpha=3 / 2)$. Although the theory was derived for the low- $\rho$ limit, ${ }^{37}$ this high value suggests that part of the foam mass hardly contributes to the stiffness (it may be contained in the nodes between the struts). Therefore, Young's modulus is highly tunable over the achieved porosity fraction of $\sim 50 \%$, enabling one-step and single-material production constructs with locally tailored properties. As an example, we printed a basic multilayer construct in which we modulated $P_{\text {air }}$ during printing, resulting in distinct regions of small cells $\left(D_{\mathrm{B}}=\right.$ $90 \pm 12 \mu \mathrm{m})$, nonporous material, and larger cells $\left(D_{\mathrm{B}}=122 \pm\right.$ $23 \mu \mathrm{m}$ ) (Figure 5). Here, the porous sections were readily

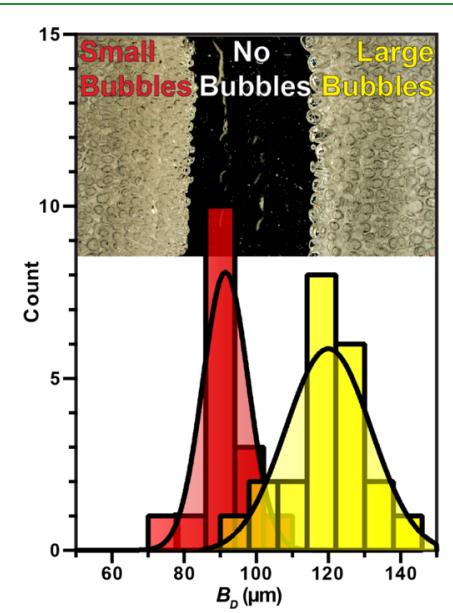

Figure 5. Printed construct with two porous regions connected by a nonporous region. The photo shows the top view of the sample. The graph depicts the bubble diameter distribution for this continuously printed multilayer porous solid. Small bubbles: $P_{\text {air }}=21.2 \mathrm{KPa}$; no bubbles: $P_{\text {air }}=18 \mathrm{KPa}$; large bubbles: $P_{\text {air }}=22.2 \mathrm{KPa}$.

bent, while the nonfoamed center section exhibited notably higher stiffness. This concept could be readily leveraged for 3D printing of functionally graded wearables such as helmets, ${ }^{1}$ where cushioning and hard protection must be integrated in a single piece.

In addition to functional gradients derived from the foam's morphology, thiol-ene inks enable the fabrication of porous materials with independently controlled chemical functionalization. As a salient consequence of the step-growth thiol-ene polymerization process, thiol-ene materials are inherently functional with thiol and alkene groups. Off-stoichiometry inks can be employed to produce materials that are predominately thiol or alkene functional materials where the functional group in excess serves as chemical handles for the modification of foams via subsequent thiol-ene reactions. ${ }^{38}$ Here, we demonstrate this ability by changing the monomer ratio of thiol to alkene within the ink to print foams comprising excess thiols, which can undergo simple postpolymerization reactions (Figure 6a). As an example, two foam filaments were printed, one from an excess thiol ink (1.2:1 SH/alkene) and another from a stoichiometric ink (1:1 SH/alkene). As indicated in Figure S4, thiol conversion plateaus at approximately $80 \%$ upon photopolymerization of inks containing $20 \mathrm{~mol} \%$ excess thiol-functional groups, whereas stoichiometric inks achieve $>95 \%$ thiol conversion. The thiol-functional and control filaments were submerged in a THF solution containing fluorescein o-methacrylate and DMPA and irradiated with 4 $\mathrm{mW} \mathrm{cm}{ }^{-2}$ UV light to ligate the fluorescein dye to the excess thiol surface. After multiple washes and gentle sonication, fluorescent microscopy revealed the dye attached efficiently to the filament containing excess thiol filament, while only faint fluorescence was observed for the control filament (Figure 6b). Similarly, thiol-functionalized and control filaments were submerged in an aqueous solution of Texas Red C2 Maleimide and trimethylamine to ligate Texas Red C2 to the foam's surface via thiol-Michael addition; again, only the filament fabricated with excess thiol in the ink showed evidence of efficient ligation (Figure 6c). Additional surface characterization of the off-stoichiometric resins via X-ray photoelectron spectroscopy (XPS) (before and after postfunctionalization with $\mathrm{CF}_{3}-\mathrm{MA}$ ) is provided in Figure S4. This demonstration confirms that simple postproduction thiol-ene reactions can successfully add new functionality to the foams.

As a final example, we demonstrate shape-memory applications at an easily accessible temperature range (Figure $7 \mathrm{a})$ enabled by the higher $T_{\mathrm{g}}\left(40{ }^{\circ} \mathrm{C}\right)$ of the cured thiol-ene inks as compared to our previously used acrylate-based inks. ${ }^{6}$ As shown in Figure $7 \mathrm{~b}$, a three-dimensional flower that deploys in water was printed on a flat surface at room temperature. Covalent cross-links within the network define the permanent shape of a flower. Subsequently, the flower was heated above $T_{\mathrm{g}}$ to $60{ }^{\circ} \mathrm{C}$ to make the material conformable. The flower was folded into a temporary shape and then cooled below its $T_{\mathrm{g}}$ to lock the temporary shape under an elastic strain. Upon contact with warm water $\left(T_{\text {water }}>T_{\mathrm{g}}\right)$, the flower unfolded into its original printed shape within $10 \mathrm{~s}$, as shown by time-stamped images in Figure S5, driven by the entropic recovery of the elastic strain within the polymer network. The mechanism for shape memory in cross-linked polymer networks using $T_{\mathrm{g}}$ as the switch is well-documented in literature, ${ }^{39-42}$ with several examples focusing on thiol-ene materials. ${ }^{43-45}$ This example highlights the one-step fabrication of shape-programmable memory foams, enabling complex patterns that save space and are deployable to predefined shapes upon application of heat. ${ }^{46}$

\section{CONCLUSIONS}

The combination of thiol-ene-based inks and direct bubble writing yielded a rapid one-step fabrication of foams with narrow cell size distributions in the absence of both surfactant and solvent. The high viscosity of the used thiol and alkene monomers yielded a viable strategy toward a surfactant-free fabrication of porous solids. In contrast, direct bubble writing with low-viscosity bubbles $(\eta \approx 10 \mathrm{mPa} \cdot \mathrm{s})$ required a surfactant to prevent their rupture prior to impacting the surface. This newfound processing window for inks with a sufficiently high viscosity to omit surfactants but with sufficiently low viscosity for bubble ejection from core-shell nozzles is highly relevant, especially since predicting and preventing the rupture of bubbles are still challenging. ${ }^{47}$ Furthermore, the lack of solvent, coupled with gelation at high thiol-ene monomer conversion, ${ }^{27-29}$ ensured that no significant shrinkage of the printed parts was observed. The use of high $T_{\mathrm{g}}$ monomer pairs in direct bubble writing was exploited in a simple shape-memory application. Additionally, altering the ratio of thiol-ene monomers allowed for the production of excess thiol foams to undergo postpolymerization modification via two different thiol-ene reactions. These examples highlight direct bubble writing as a new route toward 

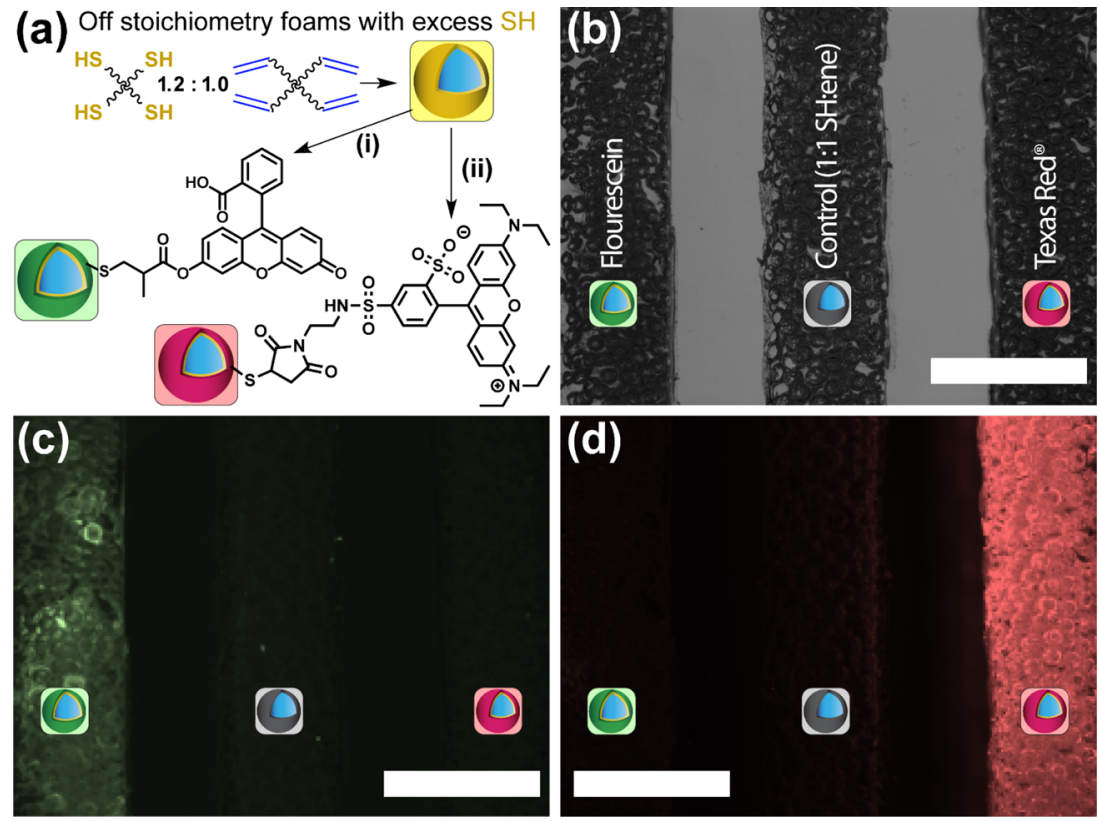

Figure 6. Off-stoichiometry resin and postfunctionalization. (a) Filaments were printed with either a thiol-to-ene ratio of 1.2:1 (yellow spheres) or a 1:1 control. After printing, the excess functional group is reacted with either (i) fluorescein $o$-methacrylate, THF, DMPA, and $4 \mathrm{~mW} \mathrm{~cm}^{-2} \mathrm{UV}$ light or (ii) Texas Red C2 Maleimide, water, and triethylamine. These reactions respectively yield materials with a green or red fluorescent functional group that is covalently bonded to the resin. (b) Optical bright-field micrograph of fluorescein labeled (left), unlabeled (middle), and Texas Red labeled (right). (c) Fluorescein labeled porous solid under 395-455 nm excitation and $480 \mathrm{~nm}$ LP filter. (d) Texas Red C2 Maleimide labeled porous solid under $540-580 \mathrm{~nm}$ excitation with a $610 \mathrm{~nm}$ LP filter. Scale bar $=4 \mathrm{~mm}$.

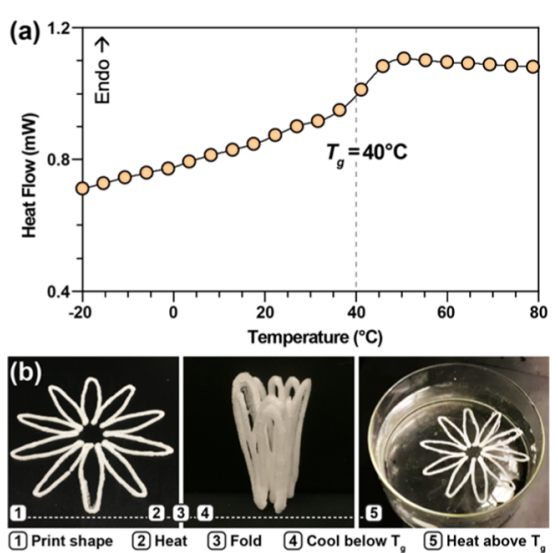

Figure 7. Thermal and shape-memory properties of a $3 \mathrm{D}$ printed porous solid. (a) Differential scanning calorimetry of cured thiol-ene foam. (b) Shape memory cycle for a 3D printed flower. The diameter of the flower is $10 \mathrm{~cm}$.

functionally graded materials with independently tunable architectural and chemical gradients.

\section{ASSOCIATED CONTENT}

\section{(s) Supporting Information}

The Supporting Information is available free of charge at https://pubs.acs.org/doi/10.1021/acsami.0c07945.

The Supporting Information includes: Figure S1. Overview of the setup; Figure 2. Time-resolved rheological properties of the resin and curing dynamics; Figure S3. Automated cell size analysis; Figure S4. Unfolding of memory foam (PDF)

\section{AUTHOR INFORMATION}

\section{Corresponding Authors}

Derek L. Patton - School of Polymer Science and Engineering, University of Southern Mississippi, Hattiesburg, Mississippi 39406, United States; 이이. orcid.org/0000-0002-8738-4750; Email: derek.patton@usm.edu

Claas Willem Visser - Engineering Fluid Dynamics Group, Thermal and Fluid Engineering Department, Faculty of Engineering Technology, University of Twente, 7500AE Enschede, The Netherlands; 이이이.org/0000-0003-31472003; Email: c.visser@utwente.nl

\section{Authors}

Dahlia N. Amato - School of Polymer Science and Engineering, University of Southern Mississippi, Hattiesburg, Mississippi 39406, United States; 이이이.org/0000-0001-9831-358X

Douglas V. Amato - School of Polymer Science and Engineering, University of Southern Mississippi, Hattiesburg, Mississippi 39406, United States

Michael Sandoz - School of Polymer Science and Engineering, University of Southern Mississippi, Hattiesburg, Mississippi 39406, United States

Jeremy Weigand - School of Polymer Science and Engineering, University of Southern Mississippi, Hattiesburg, Mississippi 39406, United States

Complete contact information is available at:

https://pubs.acs.org/10.1021/acsami.0c07945

\section{Funding}

C.W.V. acknowledges funding by the Dutch Research Council (NWO, Rubicon grant). D.N.A., D.V.A., and D.L.P. acknowledge support from the National Science Foundation (DGE1449999 and CHE-1710589). The purchase of the XPS 
instrumentation used in this work was supported by the NSF Major Research Instrumentation program (DMR-1726901).

\section{Notes}

The authors declare the following competing financial interest(s): Claas Willem Visser is co-founder and chief scientific officer of IamFluidics B.V.

\section{ACKNOWLEDGMENTS}

This research was initiated in the Lewis Lab at Harvard University. The authors thank Jennifer Lewis for fruitful discussions and Jochen Mueller for help with the nozzle design.

\section{REFERENCES}

(1) Mills, N. J.; Fitzgerald, C.; Gilchrist, A.; Verdejo, R. Polymer Foams for Personal Protection: Cushions, Shoes and Helmets. Compos. Sci. Technol. 2003, 63, 2389-2400.

(2) Rizvi, A.; Chu, R. K. M.; Lee, J. H.; Park, C. B. Superhydrophobic and Oleophilic Open-Cell Foams from Fibrillar Blends of Polypropylene and Polytetrafluoroethylene. ACS Appl. Mater. Interfaces 2014, 6, 21131-21140.

(3) Li, Z. H.; Cheng, C.; Zhan, X. Y.; Wu, Y. P.; Zhou, X. D. A Foaming process to Prepare Porous Polymer Membrane for Lithium ion Batteries. Electrochim. Acta 2009, 54, 4403-4407.

(4) Mac Murray, B. C.; An, X.; Robinson, S. S.; van Meerbeek, I. M.; O'Brien, K. W.; Zhao, H.; Shepherd, R. F. Poroelastic Foams for Simple Fabrication of Complex Soft Robots. Adv. Mater. 2015, 27, 6334-6340.

(5) Yao, H. B.; Ge, J.; Wang, C. F.; Wang, X.; Hu, W.; Zheng, Z. J.; Ni, Y.; Yu, S. H. A Flexible and Highly Pressure-sensitive Graphenepolyurethane Sponge based on Fractured Microstructure Design. Adv. Mater. 2013, 25, 6692-6698.

(6) Visser, C. W.; Amato, D. N.; Mueller, J.; Lewis, J. A. Architected Polymer Foams via Direct Bubble Writing. Adv. Mater. 2019, 31, No. 1904668.

(7) Chung, K.-y.; Mishra, N. C.; Wang, C.-c.; Lin, F.-h.; Lin, K.-h. Fabricating Scaffolds by Microfluidics. Biomicrofluidics 2009, 3, No. 022403.

(8) Loh, Q. L.; Choong, C. Three-dimensional Scaffolds for Tissue Engineering Applications: Role of Porosity and Pore Size. Tissue Eng., Part B 2013, 19, 485-502.

(9) Visser, C. W.; Kamperman, T.; Karbaat, L. P.; Lohse, D.; Karperien, M. In-air Microfluidics Enables Rapid Fabrication of Emulsions, Suspensions, and 3D modular (bio) Materials. Sci. Adv. 2018, 4, No. eaao1175.

(10) Vafai, K. Handbook of Porous Media; CRC Press, 2015.

(11) Kieback, B.; Neubrand, A.; Riedel, H. Processing Techniques for Functionally Graded Materials. Mater. Sci. Eng., A 2003, 362, 81106.

(12) Naebe, M.; Shirvanimoghaddam, K. Functionally Graded Materials: A Review of Fabrication and Properties. Appl. Mater. Today 2016, 5, 223-245.

(13) Huerre, A.; Miralles, V.; Jullien, M.-C. Bubbles and foams in microfluidics. Soft Matter 2014, 10, 6888-6902.

(14) Wang, C.-C.; Yang, K.-C.; Lin, K.-H.; Liu, Y.-L.; Liu, H.-C.; Lin, F.-H. Cartilage Regeneration in SCID Mice using a Highly Organized Three-dimensional Alginate Scaffold. Biomaterials 2012, 33, 120-127.

(15) Testouri, A.; Honorez, C.; Barillec, A.; Langevin, D.; Drenckhan, W. Highly Structured Foams from Chitosan Gels. Macromolecules 2010, 43, 6166-6173.

(16) Zhang, Y.; Choi, S. W.; Xia, Y. Modifying the Pores of an Inverse Opal Scaffold With Chitosan Microstructures for Truly Three-Dimensional Cell Culture. Macromol. Rapid Commun. 2012, 33, 296-301.

(17) Lin, J.-y.; Lin, W.-j.; Hong, W.-h.; Hung, W.-c.; Nowotarski, S. H.; Gouveia, S. M.; Cristo, I.; Lin, K.-h. Morphology and Organization of Tissue cells in 3D Microenvironment of Monodisperse Foam Scaffolds. Soft Matter 2011, 7, 10010-10016.
(18) Drenckhan, W.; Langevin, D. Monodisperse Foams in one to three Dimensions. Curr. Opin. Colloid Interface Sci. 2010, 15, 341358.

(19) Quell, A.; Elsing, J.; Drenckhan, W.; Stubenrauch, C. Monodisperse Polystyrene Foams via Microfluidics-A Novel Templating Route. Adv. Eng. Mater. 2015, 17, 604-609.

(20) Parhizkar, M.; Sofokleous, P.; Stride, E.; Edirisinghe, M. Novel Preparation of Controlled Porosity Particle/fibre Loaded Scaffolds using a hybrid Micro-fluidic and Electrohydrodynamic Technique. Biofabrication 2014, 6, No. 045010.

(21) Lo, Y.-P.; Liu, Y.-S.; Rimando, M. G.; Ho, J. H.-C.; Lin, K.-h.; Lee, O. K. Three-dimensional Spherical Spatial Boundary Conditions Differentially Regulate Osteogenic Differentiation of Mesenchymal Stromal Cells. Sci. Rep. 2016, 6, No. 21253.

(22) Costantini, M.; Colosi, C.; Mozetic, P.; Jaroszewicz, J.; Tosato, A.; Rainer, A.; Trombetta, M.; Swięszkowski, W.; Dentini, M.; Barbetta, A. Correlation between Porous Texture and Cell Seeding Efficiency of gas Foaming and Microfluidic Foaming Scaffolds. Mater. Sci. Eng., C 2016, 62, 668-677.

(23) Wang, C. C.; Yang, K. C.; Lin, K. H.; Wu, C. C.; Liu, Y. L.; Lin, F. H.; Chen, I. H. A Biomimetic Honeycomb-like Scaffold Prepared by Flow-focusing Technology for Cartilage Regeneration. Biotechnol. Bioeng. 2014, 111, 2338-2348.

(24) Ling, T.-Y.; Liu, Y.-L.; Huang, Y.-K.; Gu, S.-Y.; Chen, H.-K.; Ho, C.-C.; Tsao, P.-N.; Tung, Y.-C.; Chen, H.-W.; Cheng, C.-H.; et al. Differentiation of Lung stem/progenitor Cells into Alveolar Pneumocytes and Induction of Angiogenesis within a 3D gelatinMicrobubble Scaffold. Biomaterials 2014, 35, 5660-5669.

(25) Costantini, M.; Guzowski, J.; Żuk, P. J.; Mozetic, P.; De Panfilis, S.; Jaroszewicz, J.; Heljak, M.; Massimi, M.; Pierron, M.; Trombetta, M.; et al. Electric Field Assisted Microfluidic Platform for Generation of Tailorable Porous Microbeads as Cell Carriers for Tissue Engineering. Adv. Funct. Mater. 2018, 28, No. 1800874.

(26) Andrieux, S.; Quell, A.; Stubenrauch, C.; Drenckhan, W. Liquid Foam Templating-A Route to Tailor-made Polymer Foams. Adv. Colloid Interface Sci. 2018, 256, 276-290.

(27) Hoyle, C. E.; Bowman, C. N. Thiol-ene Click Chemistry. Angew. Chem., Int. Ed. 2010, 49, 1540-1573.

(28) Kade, M. J.; Burke, D. J.; Hawker, C. J. The Power of Thiol-ene Chemistry. J. Polym. Sci., Part A: Polym. Chem. 2010, 48, 743-750.

(29) Lowe, A. B. Thiol-ene "click" Reactions and Recent Applications in Polymer and Materials Synthesis. Polym. Chem. 2010, 1, 17-36.

(30) Childress, K. K.; Alim, M. D.; Hernandez, J. J.; Stansbury, J. W.; Bowman, C. N. Additive Manufacture of Lightly Crosslinked Semicrystalline Thiol-enes for Enhanced Mechanical Performance. Polym. Chem. 2020, 11, 39-46.

(31) Cramer, N. B.; Davies, T.; O’Brien, A. K.; Bowman, C. N. Mechanism and Modeling of a Thiol-ene Photopolymerization. Macromolecules 2003, 36, 4631-4636.

(32) Ma, S. J.; Wagner, N. J.; Kloxin, C. J. Rapid and Controlled Photo-induced Thiol-ene Wrinkle Formation via Flowcoating. Mater. Horiz. 2018, 5, 514-520.

(33) Banerji, A.; Jin, K. L.; Liu, K. W.; Mahanthappa, M. K.; Ellison, C. J. Cross-Linked Nonwoven Fibers by Room-Temperature Cure Blowing and in Situ Photopolymerization. Macromolecules 2019, 52, $6662-6672$

(34) Shanmuganathan, K.; Sankhagowit, R. K.; Iyer, P.; Ellison, C. J. Thiol-Ene Chemistry: A Greener Approach to Making Chemically and Thermally Stable Fibers. Chem. Mater. 2011, 23, 4726-4732.

(35) Esfandiari, P.; Ligon, S. C.; Lagref, J. J.; Frantz, R.; Cherkaoui, Z.; Liska, R. Efficient Stabilization of Thiol-ene Formulations in Radical Photopolymerization. J. Polym. Sci., Part A: Polym. Chem. 2013, 51, 4261-4266.

(36) Costantini, M.; Jaroszewicz, J.; Kozoń, Ł.; Szlązak, K.; Święszkowski, W.; Garstecki, P.; Stubenrauch, C.; Barbetta, A.; Guzowski, J. 3D-Printing of Functionally Graded Porous Materials Using On-Demand Reconfigurable Microfluidics. Angew. Chem., Int. Ed. 2019, 58, 7620-7625. 
(37) Gibson, L. J.; Ashby, M. F. Cellular Solids: Structure and Properties; Cambridge University Press, 1999.

(38) Amato, D. N.; Amato, D. V.; Narayanan, J.; Donovan, B. R.; Douglas, J. R.; Walley, S. E.; Flynt, A. S.; Patton, D. L. Functional, Composite Polythioether Nanoparticles via Thiol-Alkyne Photopolymerization in Miniemulsion. Chem. Commun. 2015, 51, 1091010913.

(39) Lendlein, A.; Kelch, S. Shape-memory Polymers. Angew. Chem., Int. Ed. 2002, 41, 2034-2057.

(40) Liu, C.; Qin, H.; Mather, P. T. Review of Progress in Shapememory Polymers. J. Mater. Chem. 2007, 17, 1543-1558.

(41) Xie, H.; Yang, K.-K.; Wang, Y.-Z. Photo-cross-linking: A Powerful and Versatile Strategy to Develop Shape-memory Polymers. Prog. Polym. Sci. 2019, 95, 32-64.

(42) Hasan, S. M.; Nash, L. D.; Maitland, D. J. Porous Shape Memory Polymers: Design and Applications. J. Polym. Sci., Part B: Polym. Phys. 2016, 54, 1300-1318.

(43) Nair, D. P.; Cramer, N. B.; Scott, T. F.; Bowman, C. N.; Shandas, R. Photopolymerized Thiol-ene Systems as Shape Memory Polymers. Polymer 2010, 51, 4383-4389.

(44) Michal, B. T.; Brenn, W. A.; Nguyen, B. N.; McCorkle, L. S.; Meador, M. A. B.; Rowan, S. J. Thermoresponsive Shape-Memory Aerogels from Thiol-Ene Networks. Chem. Mater. 2016, 28, 23412347.

(45) Lewis, C. L.; Meng, Y.; Anthamatten, M. Well-Defined ShapeMemory Networks with High Elastic Energy Capacity. Macromolecules 2015, 48, 4918-4926.

(46) Gladman, A. S.; Matsumoto, E. A.; Nuzzo, R. G.; Mahadevan, L.; Lewis, J. A. Biomimetic 4D printing. Nat. Mater. 2016, 15, 413418.

(47) Lhuissier, H.; Villermaux, E. Bursting Bubble Aerosols. J. Fluid Mech. 2012, 696, 5-44. 